\title{
EQUIVALENCE OF NORMS OF ANALYTICAL FUNCTIONS ON EXTERIOR OF CONVEX DOMAIN
}

\author{
N.F. ABUZYAROVA, K.P. ISAEV, R.S. YULMUKHAMETOV
}

\begin{abstract}
We study the spaces of functions holomorphic in the exterior of a bounded domain $D$ and vanishing at infinity. For each $\alpha>-\frac{1}{2}$ we introduce the integral weighted normed space $B_{2}^{\alpha}(G)$ with the weight $d^{\alpha}(z)$, where $d(z)$ denotes the distance from a point $z$ to the boundary of $G:=\mathbb{C} \backslash \bar{D}$. For $\alpha=-\frac{1}{2}$, the space $B_{2}^{\alpha}$ is chosen to be the Smirnov space. We prove that for a convex domain $D$, the norms in these spaces are equivalent to other norms defined in terms of the derivatives. For instance, the norm in the Smirnov space calculated as an integral with respect to the arc length over the boundary is equivalent to some norm defined by an integral with respect to the Lebesgue plane measure. In particular cases the proved results were obtained while studying the problem on describing the classes of Cauchy transforms of the functionals on the Bergman space on $D$. The general results may be applied in the study of Cauchy transforms of functionals on weighted Bergman spaces.
\end{abstract}

Keywords: analytic functions, Banach spaces, convex sets.

Mathematics Subject Classification: 30H05, 46E15

\section{INTRODUCTION}

Let $D$ be a bounded simply-connected Jordan domain in the complex plane and $G=\mathbb{C} \backslash \bar{D}$. By $d(\zeta), \zeta \in G$, we denote the distance from a point $\zeta$ to the boundary of $D$ :

$$
d(\zeta)=\operatorname{dist}(\zeta, \partial D)=\inf _{z \in \partial D}|\zeta-z|, \quad \zeta \in G .
$$

Let $H_{0}(G)$ be the space of functions analytic in $G$ and vanishing at infinity. For $\alpha>-\frac{1}{2}$, by $B_{2}^{\alpha}(G)$ we denote the space of the functions $\gamma \in H_{0}(G)$ with a finite norm

$$
\|\gamma\|_{\alpha}=\left(\int_{G}|\gamma(\zeta)|^{2} d^{2 \alpha}(\zeta) d v(\zeta)\right)^{\frac{1}{2}}
$$

where $d v(\zeta)$ is the area differential. For $\alpha=-\frac{1}{2}$, the space $B_{2}^{\alpha}(G)$ is identified with the Smirnov space. Without loss of generality we assume that $0 \in D$ and then the Smirnov space $E_{2}(G)$ can be defined as the completion of the space (see [1])

$$
\left\{p(\zeta) \text { is a polynomial : } p(0)=0, \int_{\partial G}\left|p\left(\frac{1}{\zeta}\right)\right|^{2} d s(\zeta)<\infty\right\},
$$

N.F. Abuzyarova, K.P. Isaev, R.S. Yulmukhametov, Equivalence of norms of analytical FUNCTIONS ON EXTERIOR OF CONVEX DOMAIN.

(C)Abuzyarova N.F., Isaev K.P., Yulmukhametov R.S. 2018.

The research of first author is supported by the grant of Russian Science Foundation (project no. 18-1100002). The second and the third authors are supported by RFBR (project no. 18-01-00095-a).

Submitted October 14, 2018. 
where $d s(\zeta)$ is the arc differential of the boundary. We introduce a natural number $n$ and by $B_{2}^{(n, \alpha)}(G)$ we denote the space of the functions $\gamma \in H_{0}(G)$ obeying $\gamma^{(n)} \in B_{2}^{\alpha}(G)$. In the space $B_{2}^{(n, \alpha)}(G)$ we consider the norm $\|\gamma\|_{n, \alpha}=\left\|\gamma^{(n)}\right\|_{\alpha}$.

The main result of the present work is the following theorem.

Theorem 1. Let $D$ be a bounded convex domain containing the origin. If $\alpha>-\frac{1}{2}$, then there exists a constant $C(\alpha)>0$ independent of the domain $D$ such that

$$
\sqrt{\frac{(\alpha+1)(2 \alpha+1)}{2}}\|\gamma\|_{n, \alpha} \leqslant\|\gamma\|_{n+1, \alpha+1} \leqslant C(\alpha)\|\gamma\|_{n, \alpha} .
$$

For $\alpha=-\frac{1}{2}$, there exists a constant $C(n)>0$ independent of the domain $D$ such that

$$
\frac{1}{2}\|\gamma\|_{n,-\frac{1}{2}} \leqslant\|\gamma\|_{n+1, \frac{1}{2}} \leqslant C(n)\|\gamma\|_{n,-\frac{1}{2}} .
$$

Thus, the spaces $B_{2}^{(n+1, \alpha+1)}(G)$ and $B_{2}^{(n, \alpha)}(G)$ coincide and the norms in these spaces are equivalent.

In particular cases, the theorem was proved in works [2], [3].

Given non-negative functions $f$ and $g$, the writing $f(x) \prec g(x), x \in X$, stands for the inequality $f(x) \leqslant C g(x), x \in X$, with some constant. The symbols $\succ$ and $\asymp$ have corresponding meanings.

\section{Proof of TheOREM 1}

Proposition 1. If the function

$$
\gamma(z)=\sum_{k=0}^{\infty} \frac{\gamma_{k}}{z^{k+1}}
$$

belongs to the space $B_{2}^{(n, \alpha)}(G)$ and $k_{\gamma}=\min \left\{k: \gamma_{k} \neq 0\right\}$, then $k_{\gamma}+n>\alpha$.

Proof. Since $d(z) \asymp|z|$ and $\left|\gamma^{(n)}(z)\right| \asymp|z|^{-\left(n+1+k_{\gamma}\right)}$ as $|z| \rightarrow \infty$, then

$$
\int_{R}^{\infty} \frac{r^{2 \alpha+1} d r}{r^{2\left(n+1+k_{\gamma}\right)}} \asymp \int_{|z|>R}\left|\gamma^{(n)}(z)\right|^{2} d^{2 \alpha} d v(z) \rightarrow 0, \quad R \rightarrow \infty,
$$

and hence, $k_{\gamma}+n>\alpha$.

Let us prove the right inequality in Theorem 1 for $\alpha>-\frac{1}{2}$. We make use of Whitney type continuation theorem (see [4]):

Theorem A. Let $F$ be an arbitrary closed set in $\mathbb{R}^{n}$. There exists a function $\delta(x)=\delta(x, F)$ defined on $\mathbb{R}^{n} \backslash F$ such that

1) the inequalities hold:

$$
c_{1} \delta(x) \leqslant \operatorname{dist}(x, F) \leqslant c_{2} \delta(x), \quad x \in \mathbb{R}^{n} \backslash F,
$$

where $c_{1}, c_{2}$ are independent of the set $F$;

2) the function $\delta(x)$ is infinitely differentiable and on $\mathbb{R}^{n} \backslash F$, the estimates hold:

$$
\left|\frac{\partial^{\beta} \delta(x)}{\partial x^{\beta}}\right| \leqslant B_{\beta}(\operatorname{dist}(x, F))^{1-|\beta|},
$$

where $B_{\beta}$ is independent of the set $F$. 
Lemma 1. Let $D$ be a bounded simply-connected Jordan domain. If $\alpha>-\frac{1}{2}, \gamma \in B_{2}^{(n, \alpha)}(G)$, then

$$
\|\gamma\|_{n+1, \alpha+1}^{2} \leqslant C(\alpha)\|\gamma\|_{n, \alpha}^{2}
$$

where $C(\alpha)$ is independent of the domain $D$.

Proof. We choose a bounded simply-connected Jordan domain $D^{\prime}$ with a smooth boundary containing the set $\bar{D}$. We apply Theorem $\mathrm{A}$ to the set $\overline{D^{\prime}}$. The obtained function $\delta(z)$ is extended by zero on $\overline{D^{\prime}}$ and is denoted by $\widetilde{\delta}(z)$. Then it follows from Theorem A that $\widetilde{\delta}^{2 \alpha} \in$ $C^{1}(\mathbb{C})$ as $\alpha>-\frac{1}{2}$. We observe that an analytic function $f$ satisfies the formula $\left|f^{\prime}\right|^{2}=\frac{1}{4} \Delta|f|^{2}$, where $\Delta=\frac{\partial^{2}}{\partial x^{2}}+\frac{\partial^{2}}{\partial y^{2}}$ is the Laplace operator. We choose a number $R$ large enough so that $\overline{D^{\prime}} \subset B(0, R)$, and by $G_{R}^{\prime}$ we denote the intersection $G^{\prime} \cap B(0, R)\left(G^{\prime}=\mathbb{C} \backslash \overline{D^{\prime}}\right)$. By the Green's formula [8] applied to the functions $\left|\gamma^{(n+1)}\right|^{2}$ and $\widetilde{\delta}^{2(\alpha+1)}$ in the domain $G_{R}^{\prime}$ we have

$$
\begin{aligned}
\int_{G_{R}^{\prime}}\left|\gamma^{(n+1)}(\zeta)\right|^{2} \widetilde{\delta}^{2(\alpha+1)}(\zeta) d v(\zeta)=\frac{1}{4} \int_{G_{R}^{\prime}} \Delta\left|\gamma^{(n)}(\zeta)\right|^{2} \widetilde{\delta}^{2(\alpha+1)}(\zeta) d v(\zeta) \\
=\frac{1}{4} \int_{\partial G_{R}^{\prime}}\left(\frac{\partial}{\partial \vec{n}}\left|\gamma^{(n)}(\zeta)\right|^{2} \delta^{2(\alpha+1)}(\zeta)-\left|\gamma^{(n)}(\zeta)\right|^{2} \frac{\partial}{\partial \vec{n}} \widetilde{\delta}^{2(\alpha+1)}(\zeta)\right) d s(\zeta) \\
+\frac{1}{4} \int_{G_{R}^{\prime}}\left|\gamma^{(n)}(\zeta)\right|^{2} \Delta \widetilde{\delta}^{2(\alpha+1)}(\zeta) d v(\zeta)
\end{aligned}
$$

Since $\widetilde{\delta} \in C^{1}$ and $\widetilde{\delta} \equiv 0$ on $\overline{G^{\prime}}$, the integrand in the first integral in the right hand side of (1) vanishes on $\partial G^{\prime}$ :

$$
\begin{aligned}
\int_{G_{R}^{\prime}}\left|\gamma^{(n+1)}(\zeta)\right|^{2} \widetilde{\delta}^{2(\alpha+1)}(\zeta) & d v(\zeta) \\
= & \frac{1}{4} \int_{|\zeta|=R}\left(\frac{\partial}{\partial \vec{n}}\left|\gamma^{(n)}(\zeta)\right|^{2} \delta^{2(\alpha+1)}(\zeta)-\left|\gamma^{(n)}(\zeta)\right|^{2} \frac{\partial}{\partial \vec{n}} \widetilde{\delta}^{2(\alpha+1)}(\zeta)\right) d s(\zeta) \\
& +\frac{1}{4} \int_{G_{R}^{\prime}}\left|\gamma^{(n)}(\zeta)\right|^{2} \Delta \widetilde{\delta}^{2(\alpha+1)}(\zeta) d v(\zeta)
\end{aligned}
$$

The relations

$$
\left|\gamma^{(n)}(\zeta)\right|^{2} \asymp R^{-2\left(n+k_{\gamma}+1\right)},\left.\left|\frac{\partial}{\partial \vec{n}}\right| \gamma^{(n)}(\zeta)\right|^{2} \mid \asymp R^{-2\left(n+k_{\gamma}\right)+3},
$$

hold true on the circumference $|\zeta|=R$ and by Theorem A,

$$
\widetilde{\delta}(\zeta)^{2(\alpha+1)} \asymp R^{2(\alpha+1)},\left|\frac{\partial}{\partial \vec{n}} \widetilde{\delta}^{2(\alpha+1)}(\zeta)\right| \asymp R^{2 \alpha+1} .
$$

Hence, as $R \rightarrow \infty$, the first integral in the right hand side in (2) tends to zero. Passing to the limit, we obtain

$$
\int_{G^{\prime}}\left|\gamma^{(n+1)}(\zeta)\right|^{2} \widetilde{\delta}^{2(\alpha+1)}(\zeta) d v(\zeta)=\frac{1}{4} \int_{G^{\prime}}\left|\gamma^{(n)}(\zeta)\right|^{2} \Delta \widetilde{\delta}^{2(\alpha+1)}(\zeta) d v(\zeta) .
$$

We apply the obtained formula to a sequence of domains $D_{m}$ with the properties:

$$
\bar{D}_{m+1} \subset D_{m}, \quad \bigcap_{m} D_{m}=\bar{D} \text {. }
$$


By the Lebesgue theorem, we can pass to the limit as $m \rightarrow \infty$ :

$$
\int_{G}\left|\gamma^{(n+1)}(\zeta)\right|^{2} \widetilde{\delta}^{2(\alpha+1)}(\zeta) d v(\zeta)=\frac{1}{4} \int_{G}\left|\gamma^{(n)}(\zeta)\right|^{2} \Delta \widetilde{\delta}^{2(\alpha+1)}(\zeta) d v(\zeta) .
$$

Estimating now $\widetilde{\delta}$ and $\Delta \widetilde{\delta}$ by Theorem A, we complete the proof of the lemma.

Let us prove the right inequality in Theorem 1 for convex domains.

We shall need the following simple properties of the function of the distance to convex domains.

Proposition 2. If $D$ is a bounded convex domain in the plane, then the distance function $d(z)=\inf \{|z-\zeta|, \zeta \in D\}, z \notin D$, possesses the properties:

1. The distance function $d(\zeta)$ is convex, in particular, is subharmonic, and satisfies the Lipschitz condition:

$$
\left|d\left(\zeta_{1}\right)-d\left(\zeta_{2}\right)\right|<\left|\zeta_{1}-\zeta_{2}\right| \text { for all } \zeta_{1}, \zeta_{2} \in G \text {. }
$$

2. The normal derivative of the distance function is identically equal to -1 . If $d(\zeta)$ is differentiable at a point $\zeta_{0}$, then $\left|\operatorname{grad} d\left(\zeta_{0}\right)\right|=1$.

3. If $D$ is a convex polygon, then $d(\zeta)$ is continuously differentiable in $G$.

Proof. 1. By $B(z, r), r>0$, we denote a ball of radius $r$ centered at a point $z$. Let $\zeta_{0} \in G$ and $3 d_{0}=\operatorname{dist}\left(\zeta_{0}, \partial D\right)$. We consider a family of straight lines $\left\{l_{\alpha}\right\}$ separating the domain $D$ from the ball $B\left(\zeta_{0}, d_{0}\right)$ and let $\left\{P_{\alpha}\right\}$ be the associated family of half-planes containing $D$. Then it is obvious that for $\zeta \in B\left(\zeta_{0}, d_{0}\right)$ we have:

$$
\operatorname{dist}(\zeta, \partial D)=\sup _{\alpha} \operatorname{dist}\left(\zeta, P_{\alpha}\right) \text {. }
$$

Since dist $\left(\zeta, P_{\alpha}\right)$ is a linear function, then $d(\zeta)=\operatorname{dist}(\zeta, \partial D)$ is a convex function. Let $\zeta_{1}, \zeta_{2} \in G$ and $z_{2} \in \partial D$ be the point of attaining the distance $d\left(\zeta_{2}\right)$, that is, $d\left(\zeta_{2}\right)=\left|z_{2}-\zeta_{2}\right|$. Then

$$
d\left(\zeta_{1}\right)-d\left(\zeta_{2}\right)=\inf _{z \in \partial D}\left|z-\zeta_{1}\right|-\left|z_{2}-\zeta_{2}\right| \leqslant\left|z_{2}-\zeta_{1}\right|-\left|z_{2}-\zeta_{2}\right| \leqslant\left|\zeta_{1}-\zeta_{2}\right| .
$$

Since $\zeta_{1}$ and $\zeta_{2}$ are of equal rights, then

$$
\left|d\left(\zeta_{1}\right)-d\left(\zeta_{2}\right)\right| \leqslant\left|\zeta_{1}-\zeta_{2}\right| .
$$

2. In view of Statement 1, the absolute values of the directional derivatives of $d(\zeta)$ do not exceed 1:

$$
\left|\frac{\partial d\left(\zeta_{0}\right)}{\partial l}\right| \leqslant 1
$$

If $z_{0} \in \partial D$ is a point of attaining the distance $d\left(\zeta_{0}\right)$, then the derivative of $d(\zeta)$ in the direction $\left(\zeta_{0}-z_{0}\right) /\left|\zeta_{0}-z_{0}\right|$ at the point $\zeta_{0}$ is equal to 1 . Since the modulus of the gradient is equal to the maximal absolute value of the directional derivatives, then $\left|\operatorname{grad} d\left(\zeta_{0}\right)\right|=1$.

3. The complement to a polygon is partitioned into half-strips, in which the distance is achieved at one of the sides of the polygon, and into the angles with the vertices at one of the vertices of the polygon. In these angles, the distance is attained at a corresponding side of the polygon. It is obvious that the distance function is continuously differentiable in the interiors of the strips and angles. It remains to check the continuous differentiability on the boundary rays. By a translation and a rotation, we overlap one of these rays with a positive part of the ordinate axis so that one of the sides of the polygon is located on the positive part of the abscissa axis. 
Let $\zeta_{0}=i y_{0}, y_{0}>0$. To the left of $\zeta_{0}$, the distance is $d(\zeta)=|\zeta|$, while to the right, the distance is $d(\zeta)=\operatorname{Im} \zeta$. If $\zeta=x+i y$, to the left of $\zeta_{0}$ we have

$$
\frac{\partial \operatorname{dist}(\zeta, D)}{\partial x}=\frac{x}{|\zeta|}, \quad \frac{\partial \operatorname{dist}(\zeta, D)}{\partial y}=\frac{y}{|\zeta|},
$$

and to the right we obtain

$$
\frac{\partial \operatorname{dist}(\zeta, D)}{\partial x} \equiv 0, \quad \frac{\partial \operatorname{dist}(\zeta, D)}{\partial y} \equiv 1
$$

Thus, we glue the first partial derivatives on the positive ordinate semi-axis.

Lemma 2. Let $D$ be a bounded convex domain containing the origin. If $\alpha>-\frac{1}{2}$, then

$$
\sqrt{\frac{(\alpha+1)(2 \alpha+1)}{2}}\|\gamma\|_{n, \alpha} \leqslant\|\gamma\|_{n+1, \alpha+1} \text {. }
$$

Proof. We choose an open convex polygon $D^{\prime} \supset \bar{D}$. We let $G^{\prime}=\mathbb{C} \backslash \bar{D}^{\prime}$ and the function dist $\left(\zeta, D^{\prime}\right), \zeta \in G^{\prime}$, is continued by zero on $\bar{D}^{\prime}$. We obtain a convex, in particular, subharmonic, in $\mathbb{C}$ function; this function is denoted by $\delta(\zeta)$.

We choose a radial smooth nonnegative mollifier $\alpha(\zeta)$ vanishing as $|\zeta| \geqslant 1$ and satisfying the condition

$$
\int_{\mathbb{C}} \alpha(\zeta) d v(\zeta)=1
$$

If a function $u(\zeta)$ is continuous in the domain $\Omega$ and

$$
u_{\varepsilon}(\zeta)=\frac{1}{\varepsilon^{2}} \int_{\mathbb{C}} \alpha\left(\frac{\zeta-z}{\varepsilon}\right) u(z) d v(z)
$$

for $\varepsilon>0$, then as $\varepsilon \rightarrow 0$, the functions $u_{\varepsilon}(\zeta)$ converges to $u(\zeta)$ uniformly on compact sets in $\Omega$ and moreover, if $u(\zeta)$ is subharmonic in $\Omega$, then $u_{\varepsilon}(\zeta)$ is also subharmonic in the domain

$$
\Omega_{\varepsilon}=\{\zeta \in \Omega: \operatorname{dist}(\zeta, \Omega)>\varepsilon\} .
$$

The properties of smooth regularizations were described in [5].

We take $\varepsilon<\operatorname{dist}\left(\partial D^{\prime}, \bar{D}\right)$ and define the regularization $\delta_{\varepsilon}(\zeta)$. The functions $\delta_{\varepsilon}(\zeta)$ are subharmonic, non-negative and $\delta_{\varepsilon}(\zeta) \equiv 0$ in the domain

$$
D_{\varepsilon}^{\prime}=\left\{\zeta \in D^{\prime}: \operatorname{dist}\left(\zeta, \partial D^{\prime}\right)>\varepsilon\right\} .
$$

It is obvious that $D_{\varepsilon}^{\prime} \subset D^{\prime}$ is a convex polygon with the sides parallel to the sides of $D^{\prime}$ and separated from the corresponding sides of $D^{\prime}$ by the distance $\varepsilon$. Under the condition $\varepsilon<$ $\operatorname{dist}\left(\partial D^{\prime}, \bar{D}\right)$, the domain $D_{\varepsilon}^{\prime}$ contains $\bar{D}$ and therefore, the function $\gamma \in H_{0}(G)$ is holomorphic on $\bar{G}_{\varepsilon}^{\prime}=\overline{\mathbb{C}} \backslash D_{\varepsilon}^{\prime}$. In the same way, as in the proof of Lemma 1 , we apply the Green's formula to the functions $\left|\gamma^{(n)}\right|^{2}, \delta_{\varepsilon}^{2(\alpha+1)}$ in the domain $G_{\varepsilon}^{\prime} \cap B(0, R)$ and then we let $R$ to tend to $\infty$. The integral over $\partial G_{\varepsilon}^{\prime}$ is equal to zero due to the function $\delta_{\varepsilon}(\zeta)$. We get:

$$
\int_{G_{\varepsilon}^{\prime}}\left|\gamma^{(n+1)}(\zeta)\right|^{2} \delta_{\varepsilon}^{2(\alpha+1)}(\zeta) d v(\zeta)=\frac{1}{4} \int_{G_{\varepsilon}^{\prime}}\left|\gamma^{(n)}(\zeta)\right|^{2} \Delta \delta_{\varepsilon}^{2(\alpha+1)}(\zeta) d v(\zeta) .
$$

By the properties of regularizations, the function $\delta_{\varepsilon}$ is subharmonic and this is why

$$
\begin{aligned}
\Delta \delta_{\varepsilon}^{2(\alpha+1)}(z) & =2(\alpha+1)(2 \alpha+1) \delta_{\varepsilon}^{2 \alpha}\left|\operatorname{grad} \delta_{\varepsilon}(\mathrm{z})\right|^{2}+2(\alpha+1) \delta_{\varepsilon}^{2 \alpha+1} \Delta \delta_{\varepsilon}(\mathrm{z}) \\
& \geqslant 2(\alpha+1)(2 \alpha+1) \delta_{\varepsilon}^{2 \alpha}\left|\operatorname{grad} \delta_{\varepsilon}(\zeta)\right|^{2}
\end{aligned}
$$


Therefore,

$$
\int_{G_{\varepsilon}^{\prime}}\left|\gamma^{(n+1)}(\zeta)\right|^{2} \delta_{\varepsilon}^{2(\alpha+1)}(\zeta) d v(\zeta) \geqslant \frac{2(\alpha+1)(2 \alpha+1)}{4} \int_{G_{\varepsilon}^{\prime}}\left|\gamma^{(n)}(\zeta)\right|^{2} \delta_{\varepsilon}^{2 \alpha}\left|\operatorname{grad} \delta_{\varepsilon}(\mathrm{z})\right|^{2} .
$$

Since $\frac{\partial u_{\varepsilon}}{\partial x}=\left(\frac{\partial u}{\partial x}\right)$, then $\left|\operatorname{grad} \delta_{\varepsilon}(\mathrm{z})\right|^{2}$ tends uniformly to $|\operatorname{grad} \delta(\mathrm{z})|^{2}$ as $\varepsilon \rightarrow 0$. Passing to the limit in relation (3), we obtain:

$$
\int_{G^{\prime}}\left|\gamma^{(n+1)}(\zeta)\right|^{2} \delta^{2(\alpha+1)}(\zeta) d v(\zeta) \geqslant \frac{2(\alpha+1)(2 \alpha+1)}{4} \int_{G^{\prime}}\left|\gamma^{(n)}(\zeta)\right|^{2} \delta^{2 \alpha}(\zeta)|\operatorname{grad} \delta(\zeta)|^{2} \mathrm{dv}(\zeta) .
$$

By Statement 2 in Proposition 2 we have $\left|\operatorname{grad} \operatorname{dist}\left(\zeta, D^{\prime}\right)\right| \equiv 1$ and hence,

$$
\int_{G^{\prime}}\left|\gamma^{(n+1)}(\zeta)\right|^{2} \delta^{2(\alpha+1)}(\zeta) d v(\zeta) \geqslant \frac{2(\alpha+1)(2 \alpha+1)}{4} \int_{G^{\prime}}\left|\gamma^{(n)}(\zeta)\right|^{2} \delta^{2 \alpha}(\zeta) d v(\zeta) .
$$

Now we choose a sequence of convex polygons contracting to $D: D_{n} \supset \bar{D}$. For each of them we write the obtained inequality and pass to the limit as $n \rightarrow \infty$.

The results of Lemmata 1 and 2 prove Theorem 1 for $\alpha>-\frac{1}{2}$. Let us prove Theorem 1 for $\alpha=-\frac{1}{2}$.

Lemma 3. If $\gamma \in E_{2}(G)$, then

$$
\frac{1}{2}\|\gamma\|_{E_{2}(G)}^{2} \leqslant\|\gamma\|_{1,1 / 2}^{2} \leqslant A(D)\|\gamma\|_{E_{2}(D)}^{2},
$$

where $A(D)$ is a constant depending on the domain $D$.

Proof. By the definition, the subspace $H_{0}(\bar{G})$ of $E_{2}(G)$ consisting of the functions analytic in $\bar{G}$ and vanishing at infinity is everywhere dense. This is why it is sufficient to prove the lemma for the functions $\gamma$ in $H_{0}(\bar{G})$. We again choose a polygon $D^{\prime} \supset \bar{D}$ and by $\delta(\zeta)$ we denote the distance function $\operatorname{dist}\left(\zeta, D^{\prime}\right)$ on $G^{\prime}=\mathbb{C} \backslash \bar{D}^{\prime}$ continued by zero on $D^{\prime}$. We choose $\varepsilon<\frac{1}{2} \operatorname{dist}\left(\partial D^{\prime}, \bar{D}\right)$ and as in the proof of Lemma 2, we introduce the regularization $\delta_{\varepsilon}(\zeta)$. By $G_{\varepsilon}$ denote the complement to the set

$$
D_{\varepsilon}=\left\{\zeta \in D^{\prime}: \operatorname{dist}\left(\zeta, \partial D^{\prime}\right) \geqslant 2 \varepsilon\right\}
$$

We observe that by condition $\varepsilon<\frac{1}{2} \operatorname{dist}\left(\partial D^{\prime}, \bar{D}\right)$, the inclusion holds: $D_{\varepsilon} \supset \bar{D}$. This is why $\gamma \in H_{0}\left(\bar{G}_{\varepsilon}\right)$. Moreover, $\delta_{\varepsilon}(\zeta) \equiv 0$ in $D_{\varepsilon}$. By the definition, $D_{\varepsilon}$ is a polygon with the sides parallel to sides of $D^{\prime}$ separated from the corresponding sides by the distance $2 \varepsilon$.

We apply the Green's formula to the function $\left|\gamma^{\prime}\right|^{2}=\frac{1}{4} \Delta|\gamma|^{2}$ and $\delta_{\varepsilon}$ in the domain $G_{\varepsilon}$. On the boundary $G_{\varepsilon}$, the function $\delta_{\varepsilon}$ and $\frac{\partial \delta_{\varepsilon}}{\partial n}$ vanish. This is why we obtain

$$
\int_{G_{\varepsilon}}\left|\gamma^{\prime}(\zeta)\right|^{2} \delta_{\varepsilon}(\zeta) d v(\zeta)=\frac{1}{4} \int_{G_{\varepsilon}}|\gamma(\zeta)|^{2} \Delta \delta_{\varepsilon}(\zeta) d v(\zeta)
$$

As $\varepsilon \rightarrow 0$, the functions $\delta_{\varepsilon}(\zeta)$ tend to $\delta(\zeta)$ uniformly on compact sets. The functions $\Delta \delta_{\varepsilon}(\zeta)$ converge weakly to the generalized function $\Delta \delta(\zeta)$ being a non-negative Borel measure associated with a subharmonic function $\delta_{\varepsilon}$.

Let $z_{1}, \ldots, z_{k}$ be the vertices of the polygon $D^{\prime}$ and $\theta_{s}, s=1,2, \ldots, k-1$, are the directions of the outward normals to the segments $\left[z_{s}, z_{s+1}\right], \theta_{k}$ is the direction of the outward to $D^{\prime}$ normal 
to segment $\left[z_{k}, z_{1}\right]$. By $l_{s}, l_{s}^{\prime}$ we denote the rays

$$
\begin{aligned}
& l_{s}=\left\{z_{s}+t e^{i \theta_{s}}, t>0\right\}, \quad s=1,2, \ldots, k, \\
& l_{s}^{\prime}=\left\{z_{s}+t e^{i \theta_{s-1}}, t>0\right\}, \quad s=2,3, \ldots, k, \\
& l_{1}^{\prime}=\left\{z_{1}+t e^{i \theta_{k}}, t>0\right\} .
\end{aligned}
$$

We denote by $V_{s}$ the acute closed angle between the rays $l_{s}, l_{s}^{\prime}$ with the vertex at the point $z_{s}$, $s=1,2, \ldots k$. By $P_{s}$ we denote the half-strips in the exterior of $D^{\prime}$ bounded by the rays $l_{s}, l_{s+1}^{\prime}$ and the segments $\left[z_{s}, z_{s+1}\right]$; here we identify $k+1$ and 1 . Then

$$
\operatorname{dist}\left(\zeta, D^{\prime}\right)= \begin{cases}\left|\zeta-z_{s}\right| & \text { as } \quad \zeta \in V_{s}, \\ \operatorname{Re}\left(\zeta-z_{s}\right) e^{-i \theta_{s}} & \text { as } \quad \zeta \in P_{s}\end{cases}
$$

It is also easy to calculate the derivatives. If $\zeta=x+i y, z_{s}=x_{s}+i y_{s}$, then

$$
\begin{gathered}
\frac{\partial}{\partial x} \operatorname{dist}\left(\zeta, D^{\prime}\right)=\left\{\begin{array}{lll}
\frac{x-x_{s}}{\left|\zeta-z_{s}\right|} & \text { as } & \zeta \in V_{s}, \\
\cos \theta_{s} & \text { as } & \zeta \in P_{s},
\end{array}\right. \\
\frac{\partial}{\partial y} \operatorname{dist}\left(\zeta, D^{\prime}\right)=\left\{\begin{array}{lll}
\frac{y-y_{s}}{\left|\zeta-z_{s}\right|} & \text { as } & \zeta \in V_{s}, \\
\sin \theta_{s} & \text { as } & \zeta \in P_{s} .
\end{array}\right.
\end{gathered}
$$

These formulae show that the first partial derivatives are continuous, the second partial derivatives are well-defined on $G^{\prime}$ and

$$
\Delta \operatorname{dist}\left(\zeta, D^{\prime}\right)=\left\{\begin{array}{cl}
\frac{1}{\left|\zeta-z_{s}\right|} & \text { if } \quad \zeta \in V_{s}, \quad \zeta \neq z_{s} \\
0 & \text { as } \quad \zeta \in P_{s} .
\end{array}\right.
$$

Together with the formulae for the first partial derivatives this implies that the support of the associated measure $\Delta \delta(\zeta)$ is located in the union of the boundary of $D^{\prime}$ and of the closed angles $\bar{V}_{s}$. At that, since the derivatives of the function $\delta$ along the outward normal to the boundary $D^{\prime}$ have the unit jump,

$$
\Delta \delta(z)=\Delta \operatorname{dist}\left(z, D^{\prime}\right)+d s(z)
$$

and

$$
\int_{G^{\prime}}\left|\gamma^{\prime}(\zeta)\right|^{2} \operatorname{dist}\left(\zeta, D^{\prime}\right) d v(\zeta)=\frac{1}{4} \int_{G^{\prime}}\left|\gamma^{\prime}(\zeta)\right|^{2} \Delta \operatorname{dist}\left(\zeta, D^{\prime}\right) d v(\zeta)+\frac{1}{4} \int_{\partial G^{\prime}}|\gamma(\zeta)|^{2} d s(\zeta)
$$

In particular,

$$
\int_{G^{\prime}}\left|\gamma^{\prime}(\zeta)\right|^{2} \operatorname{dist}\left(\zeta, D^{\prime}\right) d v(\zeta) \geqslant \frac{1}{4} \int_{\partial G^{\prime}}|\gamma(\zeta)|^{2} d s(\zeta)
$$

Thus, we have proved the left inequality in Lemma 3 for the polygon $D^{\prime}$. For the domain $D$, the needed inequality can be obtained by means of a sequence of polygons contracting to $D$.

In order to prove the right inequality in Lemma 3, let us estimate the first integral in the right hand side in (5).

We choose the straight line $L_{s}=\left\{z_{s}^{\prime}+t e^{i \varphi_{s}}, t \in(-\infty ; \infty)\right\}$ separating the domain $D$ from the angle $V_{s}$ and let $Q_{s}$ be the half-plane bounded by this line and containing the angle $V_{s}$. By Lemma 2 in work [6] we have:

$$
\int_{-\infty}^{\infty}\left|\gamma\left(z_{s}^{\prime}+t e^{i \varphi_{s}}\right)\right|^{2} d t \leqslant C(D) \int_{\partial D}|\gamma(\zeta)|^{2} d s(\zeta)
$$


Hence, the function $\gamma$ belongs to the Hardy space in the half-plane $Q_{s}$. By Carleson theorem [7], the inequality

$$
\int_{0}^{\infty}\left|\gamma\left(z_{s}+t e^{i \theta}\right)\right|^{2} d t \leqslant B \int_{-\infty}^{\infty}\left|\gamma\left(z_{s}^{\prime}+t e^{i \varphi_{s}}\right)\right|^{2} d t
$$

holds for $\theta \in\left[\theta_{s} ; \theta_{s+1}\right]$, where $B$ is an absolute constant. Two latter inequalities imply that

$$
\int_{0}^{\infty}\left|\gamma\left(z_{s}+t e^{i \theta}\right)\right|^{2} d t \leqslant B \cdot C(D) \int_{\partial D}|\gamma(\zeta)|^{2} d s(\zeta) .
$$

By formula (4) this implies:

$$
\begin{aligned}
\int_{V_{s}}|\gamma(\zeta)|^{2} \Delta \operatorname{dist}\left(\zeta, D^{\prime}\right) d v(\zeta) & =\int_{\theta_{s}}^{\theta_{s+1}} \int_{0}^{\infty}\left|\gamma\left(z_{s}+t e^{i \theta}\right)\right|^{2} \Delta \operatorname{dist}\left(z_{s}+t e^{i \theta}, D^{\prime}\right) t d t d \theta \\
& \leqslant \int_{\theta_{s}}^{\theta_{s+1}} \int_{0}^{\infty}\left|\gamma\left(z_{s}+t e^{i \theta}\right)\right|^{2} d t d \theta \\
& \leqslant B \cdot C(D)\left(\theta_{s+1}-\theta_{s}\right) \int_{\partial D}|\gamma(\zeta)|^{2} d s(\zeta) .
\end{aligned}
$$

Again by formula (4) and in view of the identity

$$
\sum_{s=1}^{k}\left(\theta_{s+1}-\theta_{s}\right)=2 \pi
$$

where $\theta_{k+1}$ is identified with $\theta_{1}+2 \pi$, we obtain:

$$
\int_{G^{\prime}}|\gamma(\zeta)|^{2} \Delta \operatorname{dist}\left(\zeta, D^{\prime}\right) d v(\zeta) \leqslant 2 \pi B \cdot C(D) \int_{\partial D}|\gamma(\zeta)|^{2} d s(\zeta) .
$$

We substitute this estimate into (5) and passing to the limit along the sequence of convex polygons, we obtain the right inequality in Lemma 3 with the constant

$$
A(D)=\sqrt{\frac{\pi B \cdot C(D)}{2}+\frac{1}{4}} .
$$

The proofs of Lemma 3 and Theorem 1 are complete.

\section{BIBLIOGRAPHY}

1. I.I. Privalov. Boundary values of analytic functions. Gostekhizdat, Moscow (1950). (in Russian).

2. V.I. Lutsenko, R. S. Yulmukhametov. "A generalization of Wiener-Paley theorem to functionals in Smirnov spaces" // Trudy MIAN. 200, 245-254 (1991). [Proc. Steklov Inst. Math. 200, 271-280 (1993).]

3. V.V. Napalkov, R.S. Yulmukhametov. "On the Cauchy transform of functionals on a Bergman space" // Matem. Sborn. 185:7, 77-86 (1994). [Russ. Acad. Sci. Sb. Math. 82:2, 327-336 (1994).]

4. E.M. Stein. Singular integrals and differentiability properties of functions. Princeton Univ. Press, Princeton. (1970).

5. L.I. Ronkin. Introduction to the theory of entire functions of several variables. Nauka, Moscow (1971). [Transl. Math. Monog. 44. Amer. Math. Soc., Providence, R.I. (1974).]

6. V.E. Kacnelson. Generalization of Wiener-Paley theorem on representation of entire functions of finite degree // Teor. Funkts. Funkts. Anal. Pril. 1, 99-100 (1965). (in Russian). 
7. J.B. Garnett. Bounded analytic functions. (English) Zbl 0469.30024 Pure Appl. Math. 96. Academic Press, New York (1981).

8. W.K. Hayman, P.B. Kennedy. Subharmonic functions. London Math. Soc. Monog. Academic Press, London (1976).

Natalia Fairbakhovna Abuzyarova, Bashkir State University,

Zaki Validi str. 32,

450074, Ufa, Russia

E-mail: abnatf@gmail.com

Konstantin Petrovich Isaev,

Institute of Mathematics,

Federal Research Center, RAS,

Chernyshevsky str. 112,

450008, Ufa, Russia

Bashkir State University,

Zaki Validi str. 32,

450074, Ufa, Russia

E-mail: orbit81@list.ru

Rinad Salavatovich Yulmukhametov,

Institute of Mathematics,

Federal Research Center, RAS,

Chernyshevsky str. 112,

450008, Ufa, Russia

Bashkir State University,

Zaki Validi str. 32,

450074, Ufa, Russia

E-mail: Yulmukhametov@mail.ru 